\title{
Analysis of clinical characteristics and risk factors in COVID-19 patients
}

\author{
Wen $\mathrm{Lu}^{1}$, Shuhui $\mathrm{Yu}^{2}$, and $\mathrm{Ke} \mathrm{Hu}^{2}$ \\ ${ }^{1}$ Rizhao People's Hospital \\ ${ }^{2}$ Renmin Hospital of Wuhan University
}

July 16, 2020

\begin{abstract}
Abstract: The aim of this study is to evaluate the clinical characteristics and outcomes in 2019 novel coronavirus patients and to help clinicians perform correct treatment and evaluate prognosis and guide the treatment. Methods: 239 patients who were diagnosed with COVID-19 were included in this study. Patients were divided into the improvement group and the death group according to their outcome (improvement or death). Clinical characteristics and laboratory parameters were collected from medical records. Continuous variables were tested by independent sample $\mathrm{T}$ test, and categorical variables were analyzed by chi-square test or Fisher exact test. Cox proportional hazard regression model was used for survival analysis in death patients. Results: Efficacy evaluation indicated that 99 patients (41.4\%) had deteriorated, and 140 patients (58.6\%) had improved. Oxygen saturation, hemoglobin levels, infection-related indicators, lymphocyte and platelets counts, C-reactive protein, serum albumin, liver and kidney function and lactate dehydrogenase in improvement group were statistically significant between the improvement and death groups. Survival analysis revealed that upper respiratory tract symptoms, hypertension, pulmonary basic diseases, high level of white blood cells, lymphocyte counts, C-reactive protein, lower serum albumin, blood urea nitrogen, were associated with mortality risk in COVID-19. Conclusion: Patients with older age, comorbidities, lower lymphocyte counts in hemogram and serum albumin, high c reactive protein level and renal dysfunction may have higher risk to death. It warned us that more attention should be given more attention on risk management in the progression of COVID-19.
\end{abstract}

\section{Analysis of clinical characteristics and risk factors in COVID-19 patients}

Lu wen ${ }^{1,3}, \mathrm{Yu}$ Shuhui $^{2}, \mathrm{Hu} \mathrm{Ke}^{3}$

1Department of respiratory disease, People's Hosptial of Rizhao, Affiliated Clinical Hospital of Jining Medical Univerity, Jining Medical University, Rizhao, Shandong, China.

2 Department of Critical Care Medicine, Renmin Hospital of Wuhan University, Wuhan, China.

Lu wen and Yu Shuhui contribute equally.

3 Division of Respiratory Disease, Renmin Hospital of Wuhan University, Wuhan, China.

Electronic address:huke-rmhospital@163.com.

BACKGROUND: The aim of this study is to evaluate the clinical characteristics and outcomes in 2019 novel coronavirus patients and to help clinicians perform correct treatment and evaluate prognosis and guide the treatment.

METHODS: 239 patients who were diagnosed with COVID-19 were included in this study. Patients were divided into the improvement group and the death group according to their outcome (improvement or death).Clinical characteristics and laboratory parameters were collected from medical records. Continuous 
variables were tested by independent sample $\mathrm{T}$ test, and categorical variables were analyzed by chi-square test or Fisher exact test. Cox proportional hazard regression model was used for survival analysis in death patients.

RESULTS: Efficacy evaluation indicated that 99 patients (41.4\%) had deteriorated, and 140 patients $(58.6 \%)$ had improved. Oxygen saturation, hemoglobin levels, infection-related indicators, lymphocyte and platelets counts, C-reactive protein, serum albumin, liver and kidney function and lactate dehydrogenase in improvement group were statistically significant between the improvement and death groups. Survival analysis revealed that upper respiratory tract symptoms, hypertension, pulmonary basic diseases, C-reactive protein, lymphocyte counts, albumin and blood urea nitrogen were associated with mortality risk in COVID19.

CONCLUSION: Patients with older age, comorbidities, lower lymphocyte counts in hemogram and serum albumin, high c reactive protein level and renal dysfunction may have higher risk to death. It warned us that more attention should be given more attention on risk management in the progression of COVID-19.

KEYWORDS: COVID-19; risk factors of death; survival probability

\section{Introduction}

Covid-19 broke out in 2019 and spread rapidly. Confirmed cases could be found in countries around the world. The novel coronavirus has a raised spike glycoprotein on its surface. The S glycopretein of the virus binds to angiotensin converting enzyme 2 of the recipient cell, which causes the virus to enter the cell. Viruses can also enter cells through membrane fusion and release nucleic acids to synthesize new viruses, causing cytopathic disease and death, thus leading to tissue and organ lesions. Novel Coronavirus is mainly related to pulmonary infection, which can involve multiple organs such as liver, spleen, cardiovascular and cerebrovascular systems, stomach and esophagus, etc. In serious cases, it can develop into acute respiratory distress syndrome, metabolic acidosis and multi-organ dysfunction syndrome, or even death. The initial clinical symptoms are mostly fever, dry cough and fatigue. A few patients are accompanied by nasal congestion, runny nose, pharyngeal pain, myalgia and diarrhea, some patients may have no obvious symptoms. Previous studies[1] suggest that the most prevalent comorbidities were hypertension(17\%),DM (8\%) and CVD (5\%) in COVID-19. It is important to evaluate patients' clinical symptoms based on current influencing factors and comorbidities. Therefore, it is necessary to evaluate possible factors that may result the progression or improvement of COVID-19 patients. Therefore, the influencing factors of status for COVID-19 patients and the result was investigated and analyzed in order to provide guidance about the treatment of this disease.

\section{Patients and Methods}

The study was approved by the the Ethics Committee of Renmin Hospital of Wuhan University (No. WDRY2020-K019). The requirement for written informed consent was waived in consideration of emerging infectious diseases. The study included data from 239 COVID-19 patients in Renmin Hospital of Wuhan University in China from December 31, 2020 to April 20, 2020. Patients diagnostic criteria about COVID19: Real-time fluorescent RT-PCR was used to detect COVID-19 in nasal or pharyngeal swab specimens, and patients with positive results twice were included in the study. In addition, relevant clinical symptoms, laboratory parameters, and major treatments were also included. The outcomes of the patients included improvement to discharge criteria or worsening to death. The patient discharge criteria and clinical types shall be based on the COVID-19 diagnosis and treatment protocol version 6 .

\section{Clinical characteristics andtherapeutic modalities}

The following clinical symptoms were recruited in the study: 1 . The upper respiratory tract symptoms such as cough, runny nose or sneezing, 2. Oppression in chest or dyspnea, 3. Muscle pain, 4. Headache, 5. Gastrointestinal symptoms such as nausea, vomiting and diarrhea, 6. Comorbidities, 7. Body temperature, 8. Time from onset to admission, 9. Total time in hospital, Comorbidities included a history of pulmonary basic disease (chronic obstructive pulmonary disease, lung cancer, bacterial pneumonia, pulmonary heart disease, et.al),hypertension, coronary artery disease, diabetes,cerebral infarction. Clinical characters include 
clinical symptoms, time from symptom onset to hospitalization, total length of hospitalization, treatment and medication, and disease outcome.

\section{Laboratory parameters}

Routine tests were performed to determine blood routine, liver and kidney function, creatinine, albumin, procalcitonin, C-reactive protein, , and potassium levels. Oxygen saturation was monitored.

\section{Statistical analysis}

SPSS.19 and R softwares statistical analysis was performed using the R Software. The measurement data were statistically described by mean \pm standard deviation and performed by $t$ test. Count data was described by frequency, and the chi-square test or Fisher's exact test was used for the comparison between groups. Multivariable analyses to identify factors associated with death from COVID-19 patients were performed by cox proportional hazards regression model. $\mathrm{P}<0.05$ was considered statistically significant.

\section{Results}

\section{Demographic characters and laboratory parameters}

The cohort consisted of 239 patients, 140 patients met discharge criteria (58.6\%) and 99(41.4\%) patients eventually returned to death (\%).Among the patients, 122(51\%) were male and 117(49\%) were female. The median age was $57(39,71)$ years. 107 patients were aged [?]60 years $(44.8 \%)$. Patients were divided into the improvement group and death groups according to their final outcomes. The majority of patients had low and moderate fever, while the remaining patients had high or no fever. A higher proportion of patients in the improvement group came to the hospital within 6 days of the onset of symptoms, as opposed to patients in the death group, who took longer than 6 days from symptom onset to treatment. The mean age of patients in the death group was significantly higher than that in the improvement group(70.00+-13.53 vs.47.47+$16.83, \mathrm{p}<0.001)$. Patients in the death group had higher rates of upper respiratory symptoms(coughing , runny nose, and sneezing), dyspnea, headache, muscle pain, gastrointestinal symptoms(vomiting and diarrhea), and comorbidities. There were statistically significant differences in hypertension, diabetes and coronary heart disease among the comorbidients. Among clinical symptoms, there were statistically significant differences in upper respiratory tract symptoms and dyspnea. The proportion of male in death group was higher than that in the improvement group, and the difference was statistically significant. All data of patients with demographic characters, clinical symptoms and comorbidities were summarized in Table1.

\section{Discussion}

Coronavirus disease 2019 (COVID-19) is the seventh known human coronavirus ${ }^{[2]}$. It is a new coronavirus caused by SARS-COV-2 coronavirus. The new virus has an envelope ${ }^{[3]}$, consisting of a single strand of RNA.Sars-cov-2 is thought to have originated in bats, with $89 \%$ to $96 \%$ of its nucleic acid sequence identical to that of the virus carried by bats ${ }^{[4]}$. SARS-CoV-2 is believed to have originated in bats, similar to many other coronaviruses, because it shares $89 \%$ to $96 \%$ nucleotide identity with bat coronaviruses. Sars-cov- 2 mainly transmitted through droplets, but can also be transmitted by aerosols. The median incubation period of the virus is $4-5$ days, and $97.5 \%$ of patients develop symptoms at 11.5 days after infection. At the start of the pandemic, The median period from clinical symptoms onset to progression to death is about 14 days ${ }^{[5]}$. Morbidity and mortality varied from time to time and region to region, for reasons that may be related to virus variation in addition to regional differences.

For its infectiousness, the number of cases increases exponentially without control measures. The hyperinflammatory response triggered by SARS-CoV-2 is the principal causes of death in infected patients ${ }^{[6]}$. The most common symptoms are fever and tussiculation, some patients appear runny nose, sneezing, a small number of patients develop nausea, vomiting or diarrhea. Part of the population can have no symptoms, or tends to improve after the infection, some people can appear rapid progression such as respiratory failure even to death ${ }^{[7]}$. The mortality increased significantly in patients with cardiovascular diseases, diabetes, COPD and hypertension ${ }^{[8]}$. Fatality increases with the progression of the severity of disease. We analyzed 
some relevant indicators and outcomes of patients diagnosed with COVID-19 in a hospital in wuhan in order to provide guidance for the treatment and prognosis of patients with coronavirus pneumonia.

The study included 239 patients with confirmed COVID-19. All patients were assessed until disease outcome( improve or dead). The study showed that 140 patients were discharged with a better health condition and 99 patients died. There was a statistically significant difference in age between the two groups, suggesting that old age is a risk factor for the disease, which is usually accompanied by a variety of complications, resulting in complicated conditions.

Data from the Chinese Center for Disease Control and Prevention showed that $80 \%$ patients experienced mild disease or even non-symptom and $20 \%$ developed severe symptoms such as respiratory failure and multiple organ failure. Previous observational studies reported patients with old age and comorbidities were more likely to deteriorate. It was later found that previously healthy patients also developed severe hypoxemia and respiratory failure, which were caused by inflammatory cascades ${ }^{[6,9]}$. Pathological examination showed lesions involved multiple organs in severe patients ${ }^{[10]}$.

Studies have shown that inflammatory markers C-reactive protein and neutrophil-to-lymphocyte ratio are risk factors in COVID patients ${ }^{[11-13]}$. Excessive inflammatory responses, including high levels of cytokines, lymphocytosis, and mononuclear macrophage infiltration, are considered important reasons for the rapid progression of the disease ${ }^{[6]}$. Our study found significant differences in white blood cell counts, neutrophil counts and lymphocyte counts between the improvement group and the death group, CRP was also associated with the risk of death, suggesting that excessive inflammation is a risk factor for patients and should be controlled clinically. Given the consideration of the importance of white blood cell and neutrophil counts for the COVID-19, we also plot the two factors in the survival curve.

In our study, survival analysis suggests that renal function factor such as blood urea nitrogenis was associated with a risk of death in patients. The differences of urea nitrogen and creatinine between the improvement group and the death group were statistically significant. Data from complete autopsy including histopathologic and virologic analysis in 12 patients who died from COVID-19 showed that high viral RNA titers were detected in kidney ${ }^{[14]}$. A study ${ }^{[15]}$ involving 701 patients with COVID-19 found that $5.1 \%$ patients experienced acute kidney injury. The mechanism involved acute tubular injury may be associated with infiltration of lymphocytes and monocytes in renal tissue. In addition, cytokine injury and organ interference may also play a role in some biological processes. Patients with renal dysfunction who are infected with SARS-CoV-2 coronavirus will face an increased risk of death, suggesting that effective renal function control should be paid attention into the clinical treatment. Serum albumin levels partially reflect body's immune state. Patients with bad nutritional status are more likely to experience lower serum albumin levels, which is harmful to the antibody production and virus clearance, and should be given high attention. Recent study suggest ${ }^{[9]}$ showed that poor nutritional status was significant risk factors for severe COVID-19 infection. Severe anorexia, and malnutrition may increase risks for respiratory failure and even required noninvasive ventilation ${ }^{[16]}$.

Assessment of the risk factors of the disease is helpful for clinicians to timely understand the risk of disease progression, so as to carry out appropriate and multifaceted intervention earlier to achieve the best therapeutic purpose. There were a few limitations in our study. Our data sources were single-center and the sample size was not large enough to represent all infected people. Highly subjective outcomes such as pain may bias the accessment results. Patients without access to treatment were not included. Some patients were complicated with uremia, resulting in a large degree of dispersion of creatinine values in samples.

\section{Funding}

This work was supported by the National Key Research and Development Plan for the Emergency Management of Novel Coronavirus Pneumonia (No. 2020YFC0845100) and the Science and Technology Key Project on Novel Coronavirus Pneumonia, Hubei province, China (project number: 2020FCA002).

\section{Reference}


1. Yang J,Zheng Y,Gou X, et al. Prevalence of comorbidities and its effects in patients infected with SARS-CoV-2: a systematic review and meta-analysis. Int J Infect Dis, 2020. 94: 91-95.

2. Lu R,Zhao X,Li J, et al. Genomic characterisation and epidemiology of 2019 novel coronavirus: implications for virus origins and receptor binding. Lancet, 2020. 395(10224): 565-574.

3. Maan S,Maan N S,Nomikou K, et al. Complete genome characterisation of a novel 26th bluetongue virus serotype from Kuwait. PLoS One, 2011. 6(10): e26147.

4. Xu X,Chen P,Wang J, et al. Evolution of the novel coronavirus from the ongoing Wuhan outbreak and modeling of its spike protein for risk of human transmission. Sci China Life Sci, 2020. 63(3): 457-460.

5. Wang W,Tang J,Wei F. Updated understanding of the outbreak of 2019 novel coronavirus (2019-nCoV) in Wuhan, China. J Med Virol, 2020. 92(4): 441-447.

6. Merad M,Martin J C. Pathological inflammation in patients with COVID-19: a key role for monocytes and macrophages. Nat Rev Immunol, 2020. 20(6): 355-362.

7. Zhang Y,Bian S,Liu X, et al. Positive Rate of Different Hepatitis B Virus Serological Markers in Peking Union Medical College Hospital, a General Tertiary Hospital in Beijing. Chin Med Sci J, 2016. 31(1): 17-12.

8. Wu Z,McGoogan J M. Characteristics of and Important Lessons From the Coronavirus Disease 2019 (COVID-19) Outbreak in China: Summary of a Report of 72314 Cases From the Chinese Center for Disease Control and Prevention. JAMA, 2020.

9. Zabetakis I,Lordan R,Norton C, et al. COVID-19: The Inflammation Link and the Role of Nutrition in Potential Mitigation. Nutrients, 2020. 12(5).

10. Zhou M,Zhang X,Qu J. Coronavirus disease 2019 (COVID-19): a clinical update. Front Med, 2020. 14(2): 126-135.

11. Wu C,Chen X,Cai Y, et al. Risk Factors Associated With Acute Respiratory Distress Syndrome and Death in Patients With Coronavirus Disease 2019 Pneumonia in Wuhan, China. JAMA Intern Med, 2020.

12. Ruan Q,Yang K,Wang W, et al. Clinical predictors of mortality due to COVID-19 based on an analysis of data of 150 patients from Wuhan, China. Intensive Care Med, 2020. 46(5): 846-848.

13. Zhou F, Yu T,Du R, et al. Clinical course and risk factors for mortality of adult inpatients with COVID-19 in Wuhan, China: a retrospective cohort study. Lancet, 2020. 395(10229): 1054-1062.

14. Bourgonje A R,Abdulle A E,Timens W, et al. Angiotensin-converting enzyme 2 (ACE2), SARS-CoV-2 and the pathophysiology of coronavirus disease 2019 (COVID-19). J Pathol, 2020.

15. Cheng Y,Luo R, Wang K, et al. Kidney disease is associated with in-hospital death of patients with COVID-19. Kidney Int, 2020. 97(5): 829-838.

16. Caccialanza R,Laviano A,Lobascio F, et al. Early nutritional supplementation in non-critically ill patients hospitalized for the 2019 novel coronavirus disease (COVID-19): Rationale and feasibility of a shared pragmatic protocol. Nutrition, 2020. 74: 110835. 
Table 1. Clinical characteristics and comorbidities in two groups of COVID-19 patients

\begin{tabular}{|c|c|c|c|c|}
\hline \multirow{2}{*}{$\begin{array}{l}\text { Demographic and } \\
\text { clinical characters }\end{array}$} & \multicolumn{2}{|c|}{ n $(\%)$} & \multirow{2}{*}{$\begin{array}{c}\text { Total } \\
(n=239)\end{array}$} & \multirow[b]{2}{*}{$\mathbf{P}$} \\
\hline & $\begin{array}{c}\text { Imp rovement } \\
(n=140)\end{array}$ & $\begin{array}{l}\text { Death } \\
(n=99)\end{array}$ & & \\
\hline \multicolumn{5}{|l|}{ Age (years) } \\
\hline$<60$ years & $106(75.7 \%)$ & $26(26.3 \%)$ & $132(55.2 \%)$ & $<0.001$ \\
\hline$>60$ years & $34(24.3 \%)$ & $73(73.7 \%)$ & $107(44.8 \%)$ & \\
\hline \multicolumn{5}{|l|}{ Sex } \\
\hline Male & $63(45 \%)$ & $59(59.6 \%)$ & $122(51.0 \%)$ & 0.035 \\
\hline Female & $77(55 \%)$ & $40(40.4 \%)$ & $117(49.0 \%)$ & \\
\hline \multicolumn{5}{|l|}{ URS } \\
\hline Yes & $77(55 \%)$ & $86(86.9 \%)$ & $163(68.2 \%)$ & $<0.001$ \\
\hline No & $63(45 \%)$ & $13(13.1 \%)$ & $76(31.8 \%)$ & \\
\hline \multicolumn{5}{|l|}{ Dyspnea } \\
\hline Yes & $53(37.9 \%)$ & $62(62.6 \%)$ & $115(46.9 \%)$ & $<0.001$ \\
\hline No & $87(62.1 \%)$ & $37(37.4 \%)$ & $124(51.9 \%)$ & \\
\hline \multicolumn{5}{|l|}{ Muscle pain } \\
\hline Yes & $32(22.9 \%)$ & $30(30.3 \%)$ & $62(25.9 \%)$ & 0.20 \\
\hline No & $108(77.1 \%)$ & $69(69.7 \%)$ & $177(74.1 \%)$ & \\
\hline \multicolumn{5}{|l|}{ Headache } \\
\hline Yes & $13(9.3 \%)$ & $11(11.1 \%)$ & $24(10.0 \%)$ & 0.667 \\
\hline No & $127(90.7 \%)$ & $88(88.9 \%)$ & $215(90.0 \%)$ & \\
\hline \multicolumn{5}{|l|}{ Pulm onary basic diseases } \\
\hline Yes & $7(5 \%)$ & $12(12.1 \%)$ & $19(7.9 \%)$ & 0.054 \\
\hline No & $133(95 \%)$ & $87(87.9 \%)$ & $220(92.1 \%)$ & \\
\hline \multicolumn{5}{|l|}{ Cerebral infarction } \\
\hline Yes & $5(3.6 \%)$ & $8(8.1 \%)$ & $13(5.4 \%)$ & 0.154 \\
\hline No & $135(96.4 \%)$ & $91(91.9 \%)$ & $226(94.6 \%)$ & \\
\hline \multicolumn{5}{|l|}{ Temperature $\left({ }^{\circ} \mathrm{C}\right)$} \\
\hline$<37.3$ & $31(22.1 \%)$ & $13(13.1 \%)$ & $44(18.4 \%)$ & \\
\hline $37.4-38$ & $27(19.3 \%)$ & $12(12.1 \%)$ & $39(16.3 \%)$ & 0.90 \\
\hline $38.1-39$ & $69(49.3 \%)$ & $64(64.6 \%)$ & $133(55.6 \%)$ & 0.06 \\
\hline$>39$ & $13(9.3 \%)$ & $10(10.1 \%)$ & $23(9.6 \%)$ & 0.68 \\
\hline \multicolumn{5}{|l|}{ Time to admission(days) } \\
\hline$>6$ & $61(43.6 \%)$ & $68(68.7 \%)$ & $129(54.0 \%)$ & $<0.001$ \\
\hline$\leqslant 6$ & $79(56.4 \%)$ & $31(31.3 \%)$ & $110(46.0 \%)$ & \\
\hline \multicolumn{5}{|l|}{ Hospital days(days) } \\
\hline$>7$ & $117(83.6 \%)$ & $26(26.3 \%)$ & $143(59.8 \%)$ & $<0.001$ \\
\hline$\leqslant 7$ & $23(16.4 \%)$ & $73(73.7 \%)$ & $96(40.2 \%)$ & \\
\hline \multicolumn{5}{|l|}{ Clinical type } \\
\hline light type & $8(5.6 \%)$ & $0(0.0 \%)$ & $8(3.3 \%)$ & 0.53 \\
\hline common-type & $127(91.4 \%)$ & $6(6.1 \%)$ & $133(55.6 \%)$ & $<0.0001$ \\
\hline severe type & $9(6.5 \%)$ & $8(8.1 \%)$ & $17(7.1 \%)$ & 0.63 \\
\hline critical type & $2(1.4 \%)$ & $85(85.8 \%)$ & $87(36.4 \%)$ & \\
\hline
\end{tabular}


Table 2. Laboratory parameters in two groups of COVID-19 patients

\begin{tabular}{|c|c|c|c|}
\hline & \multicolumn{2}{|c|}{$n=239(\%)$} & \multirow[b]{2}{*}{$\mathbf{P}$} \\
\hline & $\begin{array}{c}\text { Im provem ent } \\
(n=140)\end{array}$ & $\begin{array}{l}\text { Death } \\
(n=99)\end{array}$ & \\
\hline $\mathrm{SaO}_{2}(\%)$ & $92.43 \pm 10.13$ & $86.74 \pm 10.09$ & $<0.001$ \\
\hline Hemoglobin $(g / L)$ & $127.47 \pm 21.36$ & $121.05 \pm 22.02$ & 0.025 \\
\hline $\mathrm{WBC}\left(\times 10^{9} / \mathrm{L}\right)$ & $5.16 \pm 2.24$ & $9.27 \pm 5.79$ & $<0.001$ \\
\hline Neutrophils $\left(\times 10^{9} / \mathrm{L}\right)$ & $3.89 \pm 2.59$ & $7.03 \pm 5.41$ & $<0.001$ \\
\hline Lym phocytes $(\times 109 / L)$ & $1.08 \pm 0.57$ & $0.61 \pm 0.38$ & $<0.001$ \\
\hline P1atelet $\operatorname{count}\left(\times 10^{9} / \mathrm{L}\right)$ & $200.00 \pm 70.85$ & $165.37 \pm 93.97$ & 0.001 \\
\hline $\mathrm{C}$-reactive protein $(\mathrm{mg} / \mathrm{L})$ & $105.66 \pm 63.88$ & $21.39 \pm 20.36$ & $<0.001$ \\
\hline Serum albumin $(g / L)$ & $41.47 \pm 25.90$ & $33.05 \pm 7.71$ & 0.002 \\
\hline $\mathrm{ALP}(\mathrm{U} / \mathrm{L})$ & $60.88 \pm 26.44$ & $84.91 \pm 53.95$ & $<0.001$ \\
\hline $\operatorname{AST}(\mathrm{U} / \mathrm{L})$ & $27.74 \pm 17.07$ & $55.19 \pm 39.96$ & 0.001 \\
\hline $\mathrm{ALT}(\mathrm{U} / \mathrm{L})$ & $26.48 \pm 24.64$ & $38.73 \pm 34.04$ & $<0.001$ \\
\hline Urea(mmol/L) & $4.51 \pm 1.90$ & $9.03 \pm 5.37$ & $<0.001$ \\
\hline Creatinine $(\mathrm{g} / \mathrm{L})$ & $63.31 \pm 33.72$ & $134.56 \pm 258.12$ & 0.007 \\
\hline $\mathrm{LDH}(\mathrm{U} / \mathrm{L})$ & $230.43 \pm 105.00$ & $491.21 \pm 199.05$ & $<0.001$ \\
\hline Serum sodium $(\mathrm{mmol} / \mathrm{L})$ & $136.96 \pm 17.79$ & $139.93 \pm 15.19$ & 0.179 \\
\hline Serum potassium $(\mathrm{mmol} / \mathrm{L})$ & $3.95 \pm 0.40$ & $4.13 \pm 1.07$ & 0.112 \\
\hline
\end{tabular}



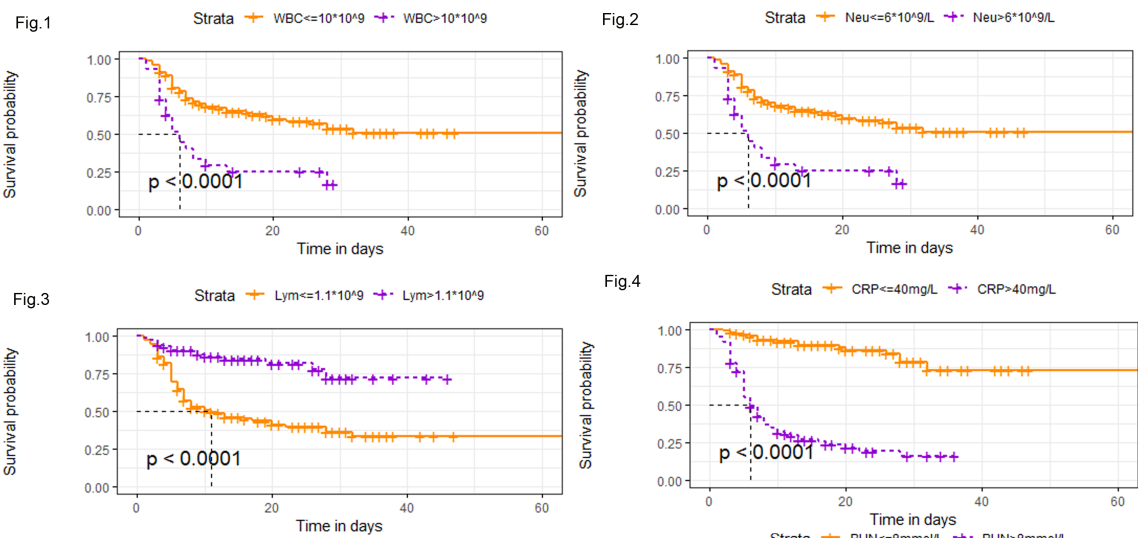

Fig. 4

Strata + CRP $<=40 \mathrm{mgl}+\cdot$ CRP $>40 \mathrm{mgh}$

Fig.

Strata + ALB $=35 g h+$ - ALB<35gh
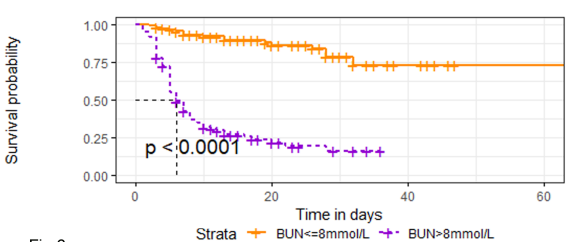

Fig.6
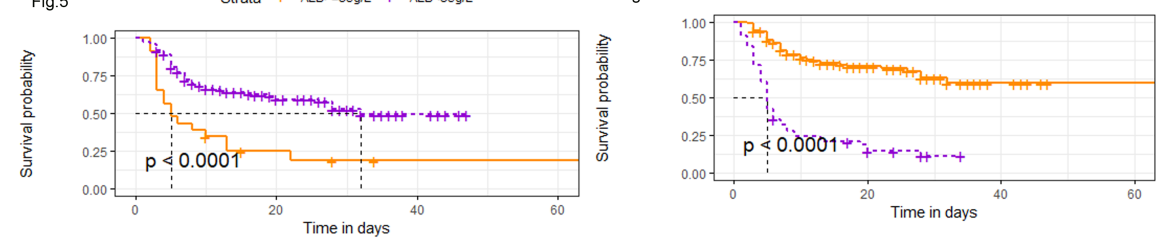

Fig.7

Strata + Age $<60$ years $+\cdot$ Age> $>60$ years

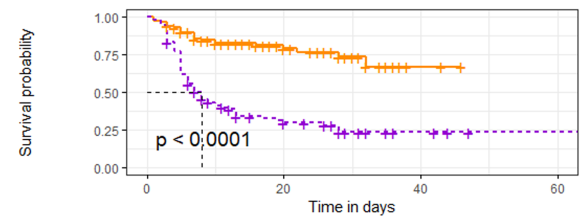

